\title{
Photoluminescent Control Ripeness of the Seeds of Plants
}

\author{
Mikhail Belyakov ${ }^{1, *}$, Elena Sokolova ${ }^{2}$, Valentina Listratenkova ${ }^{2}$, Nina Ruzanova ${ }^{2}$, and \\ Leonid Kashko ${ }^{2}$ \\ ${ }^{1}$ Branch of the national research University "MPEI" in Smolensk, Energy Passage, 1, 214013 Smolensk, \\ Russia \\ ${ }^{2}$ Smolensk State Agricultural Academy, Bolshaya Sovetskaya Str., 10/2, 214000 Smolensk Russia
}

\begin{abstract}
The development of technology for objectively determining the ripeness of plant seeds is an urgent task of modern agricultural production. An alternative to existing methods is optical photoluminescent technology, which is characterized by high accuracy, selectivity, expressiveness, as well as being remote and non-destructive. The spectral characteristics of excitation and photoluminescence of wheat, oat, and corn seeds during their maturation were measured using a spectrofluorometer using a previously developed technique. It was found that during maturation, the short-wave component of the excitation spectra decreases $(\lambda s=362 \mathrm{~nm})$ and the longwave component increases $\left(\lambda_{F}=485 \mathrm{~nm}\right)$. After measuring the luminescence spectra, the integral photoluminescence fluxes for long-wave and shortwave excitation, as well as their ratio, were determined. We have obtained statistically reliable linear regression models of the dependence of longwave and short-wave photoluminescence flows on the maturation time. Based on the obtained dependencies, a technology was developed for determining the degree of physiological maturation and making decisions about harvesting ripe seeds. It includes sample preparation, excitation and registration of luminescent radiation, amplification of the received signals and their relations, obtaining information about the degree of ripeness taking into account a priori dependencies.
\end{abstract}

\section{Introduction}

Modern innovative agriculture is in need of Express objective methods of product quality control. Due to the increasing demand for high-quality food and the changing role of man in their production, this need will increase greatly in the future. As a consequence, the need for devices that implement such methods will increase. Such methods include optical luminescent methods.

Optical methods and means of diagnostics and control are high-precision, selective, express, as well as remote and non-destructive. They have stable parameters of probing radiation, and the received signals can be accepted by radiation receivers with a wide range of characteristics, amplified and processed with the help of modern computer programs with

\footnotetext{
${ }^{1}$ Corresponding author: Bmw20100@mail.ru
} 
the issuance of complex results to support decision-making. Other advantages of optical and optoelectronic diagnostic devices are simplicity and safety of their operation, a minimum of subjective factors and the possibility of integration into existing modern agricultural machines and devices for precision agriculture.

Objective determination of seeds ripeness of plants is one of the directions in the development of agricultural electrotechnologies for the purpose of introduction of modern methods of control and support of decision-making in plant growing. Thus, the actual problem of non-destructive Express methods creation and means of multifunctional diagnostics of seed material, increasing the efficiency of agrotechnological methods of agricultural production through timely and accurate assessment of its quality parameters, requires a systematic approach. It consists in research of optical spectral properties of excitation and luminescence of seeds; theoretical and experimental substantiation of methods of diagnostics of ripeness; development of technological processes of the Express analysis of quality of seeds; creation of optoelectronic devices of measurement and control of quality of a sowing material.

The main focus of the maturation grain process is that formed in the green leaves and stem crystalline substances (sugars, amino acids, amides, etc.) are moved in dissolved form into the ear, and then into the grain. In the grain, dissolved low-molecular organic substances are converted into high-molecular insoluble spare substances (proteins, carbohydrates, fats), which fill the cells of the endosperm, aleurone layer and embryo. Maturation processes are closely related to the action of enzymes. When the grain matures, the enzyme activity gradually decreases and stabilizes at a low level characteristic of fully ripe grain.

Microscopic observations made on ripening seeds showed that as they mature, there is a gradual transformation of starch into fat ("obesity" of starch grains). The question of the substances accumulation in the ripening grain is of great practical importance in connection with the determination of the optimal timing of harvesting and reducing grain losses during harvesting.

To the date, the following methods for determining the ripeness of seeds are known:

1. Determination of ripeness of seeds by appearance and consistency. The visual method of determining the ripeness of grain is the least accurate, but, nevertheless, it is widely used in agricultural practice. This method is most often used in bad inclement weather during the ripening period, when using it requires a lot of experience and skills. The disadvantage of the method is its subjectivity.

2. Determination of seeds ripeness by weight of 1000 raw grains. The highest mass is 1000 raw grains, as studies have shown, in the phase of dough-like ripeness, about 2-3 days before the onset of wax ripeness. The disadvantages of the method are its duration and relative labor intensity.

3. Determination of grain ripeness by specific weight. From the grain after threshing, an average sample is taken and immersed in a salt solution with a specific density of $1.16 \mathrm{~g} /$ $\mathrm{cm}^{3}$. The grain in wax and full ripeness has a higher density and therefore sinks to the bottom and the grain in milk and wax ripeness floats. Thus, the grain is divided into two groups and calculate their percentage in the total mass.

4. A method for determining the ripeness of grain by staining the ear with eosin is also known. Prepare an aqueous solution of eosin at the rate of $10 \mathrm{~g} / 1$. the cut ears are immediately lowered with a straw into the solution for 3 hours. The ear is colored red in the dough-like state of the grain. With the onset of wax ripeness coloring ear stops. The disadvantage of this method is that the process of determining the grain the ripeness is long.

5. In order to reduce the time of determining the ripeness of grain by the previous method, it was proposed to use safranin as a dye. The proposed method offers to reduce the time of determining the ripeness degree of the grain from 3 hours to 15-20 minutes. 
6. On the basis of chlorophyll fluorescence, the maturity of tomato seeds is determined [1]. The system of tomato seed maturity detection based on chlorophyll fluorescence spectrum technology was developed, which includes a unit of exciting light source, a unit of fluorescent signal acquisition and a data processing unit. The excitation source unit consists of two highpower LEDs, two emitters and two DC sources and it was designed to excite the chlorophyll fluorescence of tomato seeds. The fluorescence signal detection unit consists of a QE65Pro fluorescence spectrometer, optical fiber, optical frame and narrowband filter. The detection wavelengths of the spectrometer are in the range of 200-1100 nm, the Central wavelength of the narrowband filter is $740 \mathrm{~nm}$. The fluorescent signal reception unit consists of a computer. The detection system also includes a dark camera to prevent external light and noise interference. The system test of the maturity of tomato seeds has a high accuracy of detection.

Currently used methods for determining the ripeness of seeds have significant disadvantages associated with either subjectivity or with their duration, or are destructive and require chemicals. A good alternative to the available methods is optical fluorescent, which is widely used in biomedical technologies.

Optical spectral luminescence techniques and instruments are also used in agricultural production [2-7].

To assess the hardness and soluble solids content of apples, it has been proposed to use a combination of four sensor systems (acoustic sensor, biofield hardness tester, miniature nearinfrared spectrometer and online hyperspectral scattering system) [8,9]. Fluorescent images are also used to detect rotten and damaged parts of citrus fruits [10]. The fluorescence imaging technique is widely used to measure the quality and safety of agri-food materials, in particular to detect defects in cherry tomatoes [11], automatic recognition of weeds when growing beans [12], detection of damage to wheat sprouts [13].Vis / NIR spectroscopy[14] is used to predict fruit ripeness[15,16], quality diagnostics of corn [17], potatoes [18], melons [19], apples [20], kiwis [15], olives [16, 21], citrus [22, 23], mango [24].

The purpose of this work is to study the spectral luminescent characteristics of plant seeds during their maturation and to develop a technology for photoluminescent control of the degree of ripeness of seeds.

\section{Materials and Methods}

In order to create databases to determine the ripeness of seeds and develop a decision support system for harvesting, experiments were conducted to measure the spectral luminescent characteristics of plants seeds of different ripeness.

For experiments in the growing process, batches of wheat (MIS variety), oats (Steed variety) and corn (Amatus variety) seeds with different degrees of ripeness were selected. Seeds with milk (95 days from the moment of sowing), milk-wax (103 days) and wax (109 days) ripeness were selected for wheat. Also, seeds of a later degree of maturation were selected - 4 (120 days), 5 (126 days) terms. For oats were selected seeds with milk, milkwax, wax ripeness, as well as ripe seeds (4 months). Seeds with milk ripeness ( 80 days from the sowing date), milk-wax ripeness (90 days), wax ripeness (97 days) and ripe seeds (118 days) were selected for corn.

Spectral measurements were carried out on the basis of a hardware and software complex, consisting of a multifunctional spectrofluorometer "Fluorat-02-Panorama", a computer with installed software "PanoramaPro" and an external camera for the samples under study. Spectrofluorimeter " Fluorat-02-Panorama - is a multifunctional device designed to measure the mass concentration of inorganic and organic impurities in various media, technical materials, food, biological objects in accordance with the method of measurement. The device can be used as an automatic detector in the study of excitation spectra and registration 
of luminescence, the study of photometric characteristics, phosphorescence characteristics of various objects.

For measurements with the maximum signal-to-noise ratio, a time interval is selected (measuring strobe $\tau_{\text {imp }}=20$ microseconds) and only during this time information about the signal intensity from the radiation receiver - the photoelectronic multiplier-accumulates. The scanning step was $1 \mathrm{~nm}$.

Mathematical processing of measurement results is carried out by means of the supplied software or other software products, for which the export of measurement results in ASCII and MS Excel formats is provided.

The software "PanoramaPro" provides wide opportunities in measurement of spectral characteristics of fluorescence. This is achieved, in particular, by a wide range of parameters that can be set before starting measurements. These parameters are: the spectral interval within which the scan is carried out; the level of photodetectors sensitivity; the number of pulses of the radiation source; the value of the offset during synchronous scanning.

The list of supported functions can be found in the $\mathrm{f}(\mathrm{x})$ pop-up menu. The most interesting to quantify is the integral function ( $\mathrm{f}, \mathrm{a}, \mathrm{b}$ ), where $\mathrm{f}$ is the letters of the curves; $a$ and $b$ are the start and end points of the given curve. It allows to obtain a numerical value proportional to the luminescence flux of seeds for the photoluminescence spectrum

$$
\Phi=\int_{\lambda_{1}}^{\lambda_{2}} \varphi_{1}(\lambda) \mathrm{d} \lambda
$$

$\varphi_{1}(\lambda)$ - spectral characteristics of photoluminescence,

$\lambda_{1} \ldots \lambda_{2}$ - limits of the operating spectral range of photoluminescence

and the integral value for the excitation spectrum

$$
\mathrm{H}=\int_{\lambda_{1}}^{\lambda_{2}} \eta_{e}(\lambda) \mathrm{d} \lambda
$$

$\eta_{\mathrm{e}}(\lambda)$ - the spectral characteristics of the excitation

$\lambda_{1} \ldots \lambda_{2}-$ limits of the operating spectral range of excitation.

Previously, the author established the similarity of optical luminescent properties of ripe seeds of most cereals (wheat, rye, triticale, buckwheat, oats), legumes (peas, beans) and vegetables (pepper, tomato) plants, which can be considered typical and used for the design of methods and sensors for monitoring ripeness.

\section{Results}

The results of synchronous scanning of wheat seeds of different degrees of ripeness are presented in figure 1 .

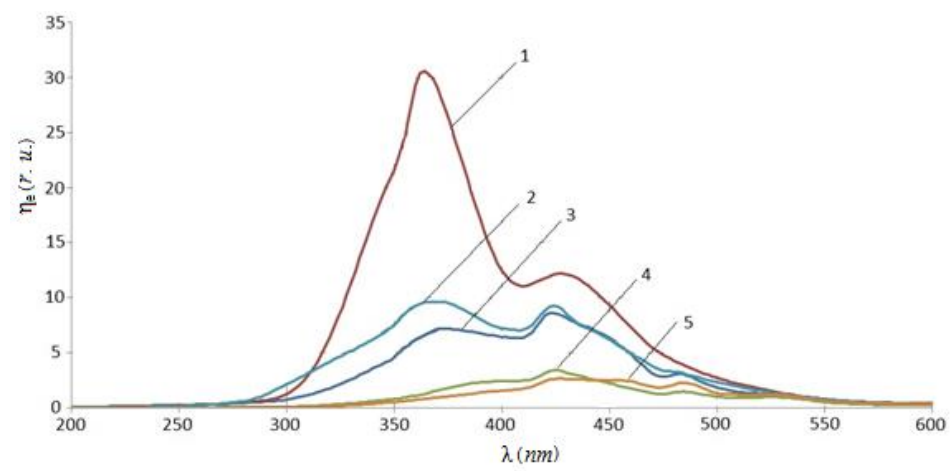


Fig. 1 Spectral characteristics of excitation during synchronous scanning for seeds: 1-milk ripeness, 2 milk-wax ripeness, 3-wax ripeness, 4-fourth term, 5-fifth term

Analyzing the curves obtained during seed maturation and seeing their qualitative changes, we can assume that this is due to changes in the concentration of various substances and complexes during maturation. The luminescence intensity of $I_{1}$ is proportional to the quantum output of $\eta$ luminescence, the intensity of the exciting light $I_{0}$, the absorption coefficient at the excitation wavelength $\varepsilon$, and the concentration of phosphor $c$

$$
I_{l}=2,3 I_{0} \varepsilon l c \eta \text {. }
$$

This equation is the mathematical basis for quantitative luminescence analysis. The dependence of the luminescence intensity on the phosphor concentration often remains linear within several orders of magnitude of the concentration. The linear dependence of the radiation intensity on the concentration is often maintained within three to four orders of magnitude of the concentration.

Panorama Pro calculated the integral values of $\mathrm{H}$ (in relative units, r. u.) in the ranges corresponding to the regions of the first $(250-410 \mathrm{~nm})$, second $(410-470 \mathrm{~nm})$ and third $(470$ $550 \mathrm{~nm}$ ) peaks.

The maxima of the obtained dependences are approximately at wavelengths $362 \mathrm{~nm}, 424$ $\mathrm{nm}, 485 \mathrm{~nm}$. Short-wave peaks of 362 and $424 \mathrm{~nm}$ are most pronounced in the excitation spectrum for seeds of milk and milk-wax ripeness. Moreover, the relative integral from the peak of $362 \mathrm{~nm}$ is the largest for the seeds of lactic ripeness, and then it decreases, decreasing at the fifth term to half of the maximum of $424 \mathrm{~nm}$. On the contrary, the peak of $485 \mathrm{~nm}$ is practically not noticeable at the first term, but by the fifth it increases its relative value by more than 2.3 times.

Similar results were obtained for oat and corn seeds.

The maxima of the obtained $\eta_{\mathrm{e}}(\lambda)$ dependences are approximately at the wavelength of $362 \mathrm{~nm}$. It can be seen that with the maturation of seeds, the maxima at the wavelength of $362 \mathrm{~nm}$ decrease.

Next, the integral values of $\mathrm{H}$ were calculated by the formula (2) in the ranges corresponding to the regions of $260-310 \mathrm{~nm}, 320-400 \mathrm{~nm}, 400-450 \mathrm{~nm}, 470-500 \mathrm{~nm}$.

In the range of $260-310 \mathrm{~nm}\left(\lambda_{\max }=290 \mathrm{~nm}\right)$ on the $80^{\text {th }}$ and $94^{\text {th }}$ days there is a peak of 68 r.u. and 94 r.u. This peak is present in $40 \%$ of milk seeds and $50 \%$ of seeds in wax ripeness. In the range of $320-400 \mathrm{~nm}\left(\lambda_{\max }=362 \mathrm{~nm}\right) \mathrm{H}$ decreases 5 times, in the range of $470-500 \mathrm{~nm}$ $\left(\lambda_{\max }=485 \mathrm{~nm}\right)$ luminescence is practically not observed.

Excitation and luminescence spectra of selected seeds of each ripeness were measured. According to the results of measurements, statistical processing was carried out, where averaging over 30 spectra was carried out [25].

The results for milk-ripe and fully-ripe wheat seeds are shown in figure 2 .

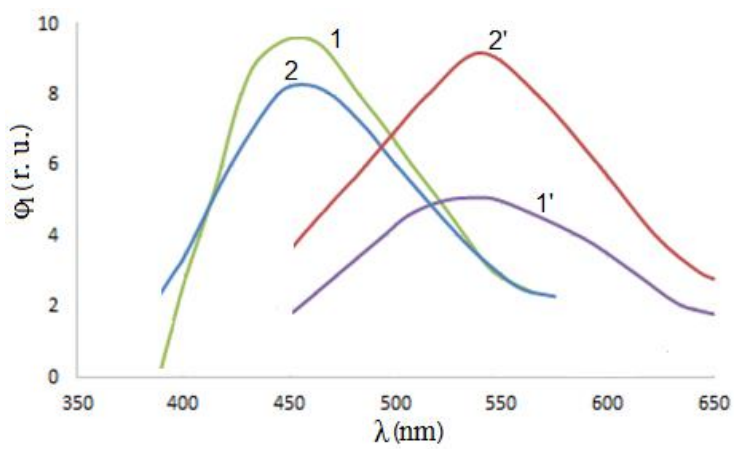


Fig. 2. Luminescence Spectra of wheat seeds of milk ripeness (1 and 1') and fully ripe (2 and 2') of short and long wavelength ranges.

Short-wave $\left(\Phi_{l}\right)$ and long-wave $\left(\Phi_{s}\right)$ photoluminescence fluxes and their ratio are calculated. The results are presented in table 1 and figure 3 .

Table 1. Results of photoluminescence flux calculation of wheat seeds of different maturity dates

\begin{tabular}{|c|c|c|c|c|}
\hline Duration & Ripening time $\boldsymbol{t}$, day & $\boldsymbol{\Phi}_{\mathbf{s}}$, r.u. & $\boldsymbol{\Phi}_{\boldsymbol{l}}$, r.u. & $\boldsymbol{\Phi}_{\mathbf{l}}$ / $\boldsymbol{\Phi}_{\mathbf{s}}$, r.u. \\
\hline 1 & 95 & 1116 & 658 & 0.59 \\
\hline 2 & 103 & 1278 & 921 & 0.72 \\
\hline 3 & 109 & 1491 & 1297 & 0.87 \\
\hline 4 & 120 & 1125 & 996 & 0.89 \\
\hline 5 & 126 & 1023 & 1114 & 1.09 \\
\hline
\end{tabular}

Equation of linear approximation of inverse dependence of flow ratio on time:

$$
t=63.76 \frac{\Phi_{1}}{\Phi_{\mathrm{s}}}+57.56
$$

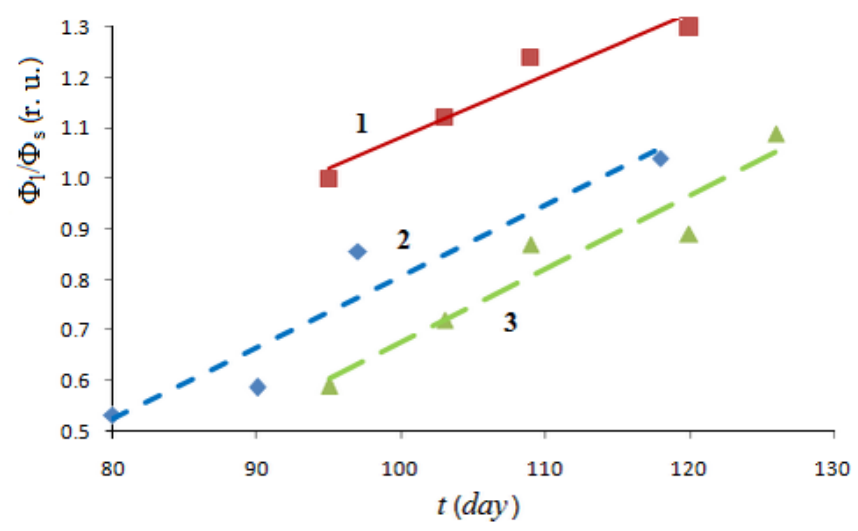

Fig. 3. Graphs of dependencies of the ratio of long-wave flows to short-wave flows on time in days: 1 - for oats, 2-for corn, 3-for wheat

Similar results for oat seeds are shown in table 2 [26].

Table 2. Results of photoluminescence flux calculation oat seeds

\begin{tabular}{|c|c|c|c|c|}
\hline Duration & Ripening time $\boldsymbol{t}$, day & $\boldsymbol{\Phi}_{\mathbf{s}}$, r.u. & $\boldsymbol{\Phi}_{\boldsymbol{l}}$, r.u. & $\boldsymbol{\Phi}_{\mathbf{l}} / \boldsymbol{\Phi}_{\mathbf{s}}$, r.u. \\
\hline 1 & 95 & 993 & 991 & 1.00 \\
\hline 2 & 103 & 983 & 1101 & 1.12 \\
\hline 3 & 109 & 913 & 1132 & 1.24 \\
\hline 4 & 120 & 913 & 1184 & 1.30 \\
\hline
\end{tabular}

Approximation equation for oat seeds:

$$
t=76.55 \frac{\Phi_{1}}{\Phi_{\mathrm{s}}}+17.57
$$

For maize seeds, spectral luminescence characteristics of $\varphi_{1}(\lambda)$ in seeds of each ripeness were measured at $\lambda=362 \mathrm{~nm}$. 
Approximation equation for corn seeds

$$
t=64.54 \frac{\Phi_{1}}{\Phi_{\mathrm{s}}}+47.58
$$

The dependencies of the flow ratios on the maturation time are shown in figure 3 .

As it can be seen from the tables and graphs with increasing ripeness of seeds, the ratio of long-wave flow to short-wave increases. The dependences are statistically reliably linearly approximated with the coefficient of determination $R^{2}$ equal to 0.9201 for wheat, 0.9355 for oats and 0.9102 for corn. These dependences (4-6) can be used to determine the degree of physiological maturity of seeds. Thus, wheat and corn seeds can be considered ripe at a ratio of $\Phi_{1} / \Phi_{\mathrm{s}}$ exceeding 1.0, and oat seeds-1.3.

The information system of Express diagnostics of level of physiological maturity of seeds allowing to define at what stage of maturation the seed was developed. Figure 4 shows its block diagram.

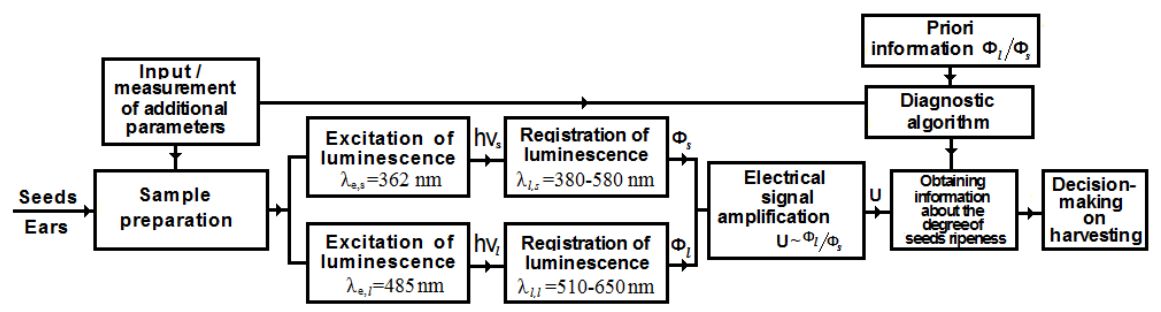

Fig. 4. Block diagram of the information system of rapid diagnostics of seeds during ripening

1. For a study with multiple plants, select a few seeds. Non-destructive testing of seeds of a full ear is possible, without human participation with the use of unmanned aerial vehicles. The test sample is placed in a dark light-tight chamber.

2. At the same stage, the seed type (culture, variety) is identified and other seed parameters, such as moisture, necessary to establish the appropriate diagnostic algorithm can be measured.

3. To excite photoluminescence, seeds are irradiated with radiation with maxima at wavelengths $\lambda_{\mathrm{e}, \mathrm{s}}=362 \mathrm{~nm}$ and $\lambda_{\mathrm{e}, \mathrm{l}}=485 \mathrm{~nm}$ (or as close to them as possible).

4. Luminescence is also recorded in two ranges: short-wave $\lambda_{1, \mathrm{~s}}=380-580 \mathrm{~nm}$ and long-wave $\lambda_{1,1}=510-650 \mathrm{~nm}$. At the output, $\Phi_{\mathrm{s}}$ and $\Phi_{1}$ luminescence streams are obtained, respectively. 5. At the next stage, the electric photo signal proportional to the ratio of $U \sim \Phi_{1} / \Phi_{\mathrm{s}}$ flows is amplified.

6. The obtained result is processed taking into account a priori information about the dependence of $\Phi_{1} / \Phi_{\mathrm{s}}$ on the ripening time (a priori database) and determined at what stage of ripeness the seeds are.

7. On the basis of the results obtained, a decision is made on the timing of harvesting.

To implement the information system, the design was developed and the main nodes of the sensor for objective rapid analysis of the degree of physiological maturity of seeds were selected (figure 5).

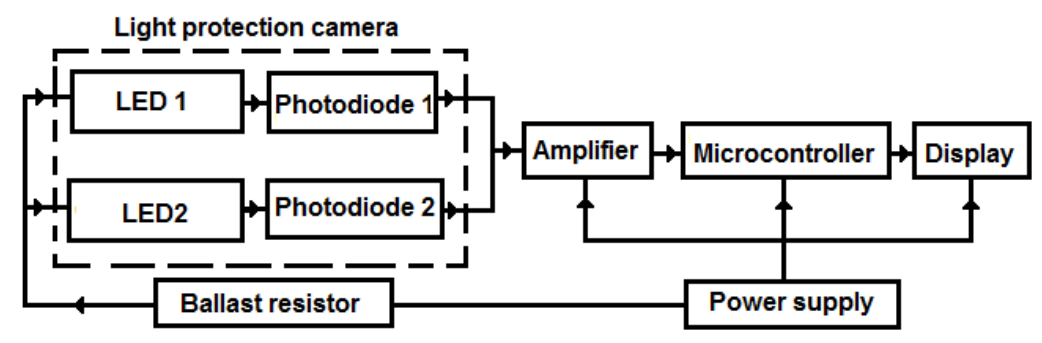


Fig. 5. Block diagram of an optoelectronic sensor for determining the ripeness of seeds

Conventionally, this block diagram can be divided into a light-optical unit and an electronic unit. The light-optical unit consists of a lighting part (light-emitting diodes are the sources of radiation) and a photodetector (photodiodes are used as photodetectors). In turn, the electronic unit consists of a signal amplifier, a microcontroller, which performs information processing according to a given algorithm, and an indicator that serves to visualize the data obtained.

The developed sensor for determining the ripeness of seeds is actually a single-chamber and is a device enclosed in a rectangular case, on the inner surface of which a black nonreflective coating is applied. The functional diagram of the sensor is shown in figure 6 .

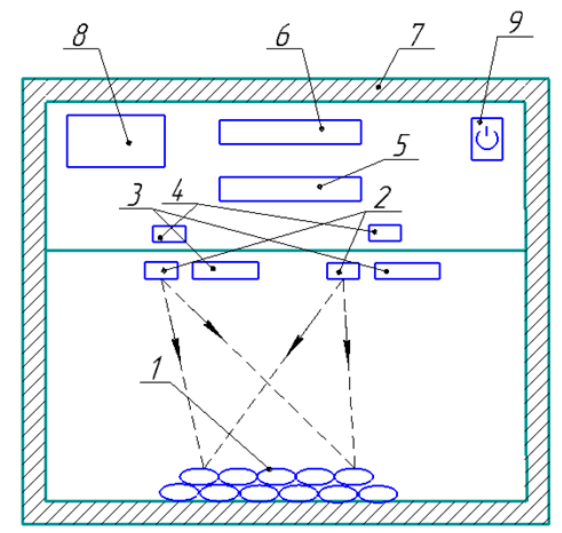

Fig. 6. Functional diagram of the sensor determine the degree of ripeness of the seeds: 1 - the investigated material; 2 - light source; 3 - detector; 4 - amplifier of the analog signal; 5 microcontroller; 6 - rendering device; 7 - the device casing; 8 - the battery; 9 - control system.

The proposed functional scheme has a number of advantages, including the absence of optical components (lenses, collimators, flat mirrors), which eliminates the need for alignment. Also, the advantages of this scheme are compact, low weight, cost, ease of setup and use of the device.

The studied samples of agricultural crops are laid out on a special table and illuminated by LEDs 2, the wavelength of which is selected taking into account the experiments done earlier, the signal enters the photodetector 3, after which it is amplified by an analog signal amplifier 4 and the processing of this signal begins on the programmed microcontroller 5 . The result obtained with the help of the visualization device 6 is delivered directly to the operator.

For the sensor being developed, it is important that there are as few attachments as possible, as it is planned to be used for rapid analysis, therefore, it should be easy to carry, compact and easy to operate. On the basis of the above structural and functional schemes was developed circuit diagram.

The main criterion for choosing a radiation source is the correspondence of its radiation spectrum to the sensitivity spectrum of seeds - the luminescence excitation spectrum. This results in the maximum efficiency - the effective return of the $\eta_{\text {eff }}$ source.

$$
\eta_{\text {eff }}=\frac{\Phi_{\text {eff }}}{\Phi_{\text {int }}}=\frac{\int_{0}^{\infty} \varphi(\lambda) S(\lambda) \mathrm{d} \lambda}{\int_{0}^{\infty} \varphi(\lambda) \mathrm{d} \lambda},
$$


where $\Phi_{\text {eff }}$ is the effective flow;

$\Phi_{\text {int }}-$ full light flow;

$S(\lambda)$ - spectral sensitivity of the receiver;

$\varphi(\lambda)$ - radiation spectrum of the source.

\section{Conclusion}

Optical photoluminescent methods for assessing the quality of plant seeds are non-contact, fast-acting, selective and often non-destructive. On their basis, it is possible to create an information system to support the decision on harvesting. The seeds of the studied cereal plants are characterized by excitation of photoluminescence in the ranges with maxima at wavelengths of $362 \mathrm{~nm}, 424 \mathrm{~nm}$ and $485 \mathrm{~nm}$. During the ripening of seeds of grain plants (for example, wheat, oats and corn), the ratio of their excitation levels and luminescence flows changes: for immature seeds, short-wave luminescence is characteristic, and long-wave luminescence prevails in ripe seeds. The dependences of the ratio of photoluminescence fluxes of the long and short wavelength ranges on the maturation time are increasing and can be statistically reliably approximated by the linear functions necessary to create a mobile database. On the basis of the obtained results, a sensor was developed to determine the level of physiological maturity of seeds, allowing by irradiating seeds with two sources with certain wavelengths and registering the photoluminescence flux with appropriate receivers, to determine at what stage of ripening the seed is.

\section{References}

1. C. Li , X. Wang, Z. Meng, Tomato seeds maturity detection system based on chlorophyll fluorescence. Proc. SPIE 10021, Optical Design and Testing VII, 1002125 (2016) doi:10.1117/12.2247866

2. M. Kaszczuk, Z. Mierczyk, M. Zygmunt, Multispectral laser scanning in plants condition analysis. Proc. SPIE 10974, Laser Technology 2018: Progress and Applications of Lasers, 109740E doi: 10.1117/12.2516763

3. X. Wang, C. Li, Z. Meng, P. Fan, J. Cai, Pepper seed variety identification based on visible/near-infrared spectral technology. Proc. SPIE 10030, Infrared, Millimeter-Wave, and Terahertz Technologies IV, 100302N (2016) doi:10.1117/12.2247569

4. Y. Lu, R. Li, R. Lu, Detection of fresh bruises in apples by structured-illumination reflectance imaging. Proc. SPIE 9864, Sensing for Agriculture and Food Quality and Safety VIII, 986406 (2016) doi:10.1117/12.2225148

5. C. Lü, X. Jiang, X. Zhou, Y. Zhang, N. Zhang, C. Wei, W. Mao, Variable selection based near infrared spectroscopy quantitative and qualitative analysis on wheat wet gluten. Proc. SPIE 10461, AOPC 2017: Optical Spectroscopy and Imaging, 1046104 (2017) doi:10.1117/12.2281441

6. X. Song, J. Wang, X. Gu, X. Xu, Winter wheat GPC estimation with fluorescence-based sensor measurements of canopy. Proc. SPIE 9637, Remote Sensing for Agriculture, Ecosystems, and Hydrology XVII, 96371L (2015) doi: 10.1117/12.2195289

7. J. Zhao, Y. Peng, S. Dhakal, L. Zhang, A. Sasao, A noninvasive technique for real-time detection of bruises in apple surface based on machine vision. Proc. SPIE 8721, Sensing for Agriculture and Food Quality and Safety V, 87210 (2013) doi:10.1117/12.2015897

8. F. Mendoza, R. Lu, H. Cen, Multi-sensor data fusion for improved prediction of apple fruit firmness and soluble solids content. Proc. SPIE 8027, Sensing for Agriculture and Food Quality and Safety III, 80270M (2011) doi: 10.1117/12.883649 
9. D. Feng, J-W. Ji, L. Zhang, S.-J. Liu, Y-W. Tian, The research development of hyperspectral imaging in apple nondestructive detection and grading. Proc. SPIE 10156, Hyperspectral Remote Sensing Applications and Environmental Monitoring and Safety Testing Technology, 101560V (2016) doi:10.1117/12.2246537

10. M.A. Momin, N. Kondo, M. Kuramoto, Y. Ogawa, T. Shigi, Study on excitation and fluorescence spectrums of Japanese citruses to construct machine vision systems for acquiring fluorescent images. Proc. SPIE 8027, Sensing for Agriculture and Food Quality and Safety III, 80270R (2011) doi: 10.1117/12.885640

11. I. Baek, B. Cho, M. Kim, Y. Kim, Determination of optimal excitation and emission wavebands for detection of defect cherry tomato by using fluorescence emission and excitation matrix. Proc. SPIE 8721, Sensing for Agriculture and Food Quality and Safety V. 872108 (2013) doi:10.1117/12.2018543

12. W.-H. Su, S. Fennimore, D. Slaughter, Fluorescence imaging for rapid monitoring of translocation behaviour of systemic markers in snap beans for automated crop/weed discrimination. Biosystems Engineering 186, 156-167 (2019) https://doi.org/10.1016/j.biosystemseng.2019.07.009

13. J. Barbedo, E. Guarienti, C. Tibola, Detection of sprout damage in wheat kernels using NIR hyperspectral imaging Biosystems Engineering 175, 124-132 (2018) https://doi.org/10.1016/j.biosystemseng.2018.09.012

14. X. Xu, L. Xie, Y. Ying, Factors influencing near infrared spectroscopy analysis of agroproducts: a review [J]. Front. Agr. Sci. Eng. 6(2), 105-115 (2019) https://doi.org/10.15302/J-FASE-2019255

15. D. Liu, W. Guo, Q. Li, D. Xie, Relationship of the bulk optical properties in 950$1650 \mathrm{~nm}$ wavelength range with internal quality and microstructure of kiwifruit during $\begin{array}{lllll}\text { maturation. Biosystems } & \text { Engineering } & \text { 184, 2019) }\end{array}$ doi:10.1016/j.biosystemseng.2019.05.005

16. A. Casson, R. Beghi, V. Giovenzana, I. Fiorindo, A. Tugnolo, R. Guidetti, Environmental advantages of visible and near infrared spectroscopy for the prediction of intact olive ripeness. Biosystems Engineering 189, 1-10 (2020). doi:10.1016/j.biosystemseng.2019.11.003

17. L. Yu, et al., Non-destructive identification of maize haploid seeds using nonlinear analysis method based on their near-infrared spectra. Biosystems Engineering 172, 144153 (2018)

18. P.-S. Liang, Nondestructive detection of zebra chip disease in potatoes using nearinfrared spectroscopy Biosystems Engineering 166, 161-169 (2018) https://doi.org/10.1016/j.biosystemseng.2017.11.019

19. M. Li, D. Han, W. Liu, Non-destructive measurement of soluble solids content of three melon cultivars using portable visible/near infrared spectroscopy Biosystems Engineering 188, 31-39 (2019). https://doi.org/10.1016/j.biosystemseng.2019.10.003

20. J. Gené-Mola, et al., Fruit detection in an apple orchard using a mobile terrestrial laser $\begin{array}{lllll}\text { scanner Biosystems } & \text { Engineering 187, 171-184 }\end{array}$ https://doi.org/10.1016/j.biosystemseng.2019.08.017.

21. V. Giovenzana, et al., Use of visible and near infrared spectroscopy with a view to online evaluation of oil content during olive processing Biosystems Engineering 172, 102109 (2018) https://doi.org/10.1016/j.biosystemseng.2018.06.001.

22. S. Moomkesh, S. Mireei, M. Sadeghi, M. Nazeri, Early detection of freezing damage in sweet lemons using Vis/SWNIR spectroscopy Biosystems Engineering 164, 157-170 (2017) https://doi.org/10.1016/j.biosystemseng.2017.10.009 
23. X. Fu, X. Wang, X. Rao, An LED-based spectrally tuneable light source for visible and near-infrared spectroscopy analysis: A case study for sugar content estimation of citrus Biosystems $\quad$ Engineering $\quad 163$ 87-93 (2017) https://doi.org/10.1016/j.biosystemseng.2017.08.022

24. V. Cortés, et al., Integration of simultaneous tactile sensing and visible and near-infrared reflectance spectroscopy in a robot gripper for mango quality assessment Biosystems Engineering 162, 112-123 (2017) https://doi.org/10.1016/j.biosystemseng.2017.08.005

25. M. V. Belyakov, V. O. Bulatikova, V. V. Dimkova, A. G. Dymnikova, Photoluminescent characteristics wheat seeds of different ripeness. XXXIII International scientific and practical conference «International scientific review of the problems and prospects of modern science and education». Boston, USA, International scientific review 4(35), 27 31 (2017)

26. V. M. Belyakov, Photoluminescence monitoring of ripeness of seeds of grain during the ripening process. Inzhenernye tekhnologii i sistemy 2, 306-319 (2019) doi: 10.15507/2658-4123.029.201902.306-319 